\title{
水分和养分添加对羊草功能性状和地上生物量的 影响
}

\author{
赵丹丹 ${ }^{1,2}$ 马红媛 ${ }^{*}$ 李 阳 $^{1,2}$ 魏继平 ${ }^{1}$ 王志春 ${ }^{1}$ \\ ${ }^{1}$ 中国科学院东北地理与农业生态研究所, 长春 130102 ; $^{2}$ 中国科学院大学, 北京 100049
}

\begin{abstract}
摘 要 研究水分和养分添加对植物功能性状的影响, 对于揭示植物对环境变化的响应和适应规律至关重要。该文采用盆栽 试验的方法, 进行不同水平水分处理(增水 $50 \%$, 减水 $50 \%$, 以 $498 \mathrm{~mm}$ 降水量作为对照)和养分添加(无养分添加, 单施氮肥, 单施磷肥, 氮磷共施), 研究羊草(Leymus chinensis)的 10 种功能性状和地上生物量对水分和养分添加的响应。得出以下结论: (1)双因素方差分析结果表明, 水分主效应对羊草株高、分菜数、茎生物量、叶生物量、叶面积、叶质量、净光合速率、蒸腾 速率、水分利用效率存在显著影响; 养分主效应对羊草分葉数、茎生物量、净光合速率、蒸腾速率、水分利用效率存在显著 影响; 水分和养分的交互作用对羊草分藥数、茎生物量、蒸滕速率、水分利用效率存在显著影响。(2)各功能性状对降水量的 响应在不同养分添加水平是不同的, 分藥数和叶面积在单施氮肥和氮磷共施条件下随降水量增加而增加, 而在无养分添加和 单施磷肥条件下无显著变化; 茎生物量在无养分添加、单施氮肥和单施磷肥条件下随降水量增加而增加, 而在氮磷共施条件 下无增加趋势; 比叶面积在单施氮肥条件下增水处理显著低于对照组, 而在其他养分添加条件下无明显变化。(3)短期氮磷处 理显著影响羊草叶片光合生理性状, 而对叶形态性状影响不显著。(4)羊草地上生物量随降水量的增加呈现上升趋势, 并且在 单施氮肥条件下, 增水处理使地上生物量达到最高, 为 $522.55 \mathrm{~g} \cdot \mathrm{m}^{-2}$ 。总之, 羊草的功能性状对降水量增加表现出明显的响应, 响应格局在不同养分条件下不同, 反映了其对水肥环境变化的适应。
\end{abstract}

关键词 植物功能性状; 形态性状; 生理性状; 地上生物量

赵丹丹, 马红媛, 李阳, 魏继平, 王志春 (2019). 水分和养分添加对羊草功能性状和地上生物量的影响. 植物生态学报, 43, 501-511. DOI: $10.17521 /$ cjpe.2019.0041

\section{Effects of water and nutrient additions on functional traits and aboveground biomass of Ley- mus chinensis}

ZHAO Dan-Dan ${ }^{1,2}$, MA Hong-Yuan ${ }^{1 *}$, LI Yang ${ }^{1,2}$, WEI Ji-Ping ${ }^{1}$, and WANG Zhi-Chun ${ }^{1}$

${ }^{1}$ Northeast Institute of Geography and Agroecology, Chinese Academy of Sciences, Changchun 130102, China; and ${ }^{2}$ University of Chinese Academy of Science, Beijing 100049, China

\section{Abstract}

Aims The research on the response of plant functional traits to environmental change, such as precipitation change and nutrient additions is very important to understand how plant species adapt to variable environments.

Methods We conducted a pot experiment with a gradient of water treatments (increase precipitation by $50 \%$, HW; decrease precipitation by $50 \%$, LW; take $498 \mathrm{~mm}$ precipitation as control, MW) and nutrient additions (without nutrient addition, $\mathrm{CK}$; nitrogen $(\mathrm{N})$ addition, NA; phosphorus $(\mathrm{P})$ addition, $\mathrm{PA}$; nitrogen and phosphorus additions, $\mathrm{N}+\mathrm{P}$ ). We investigated 11 plant functional traits and aboveground biomass of Leymus chinensis.

Important findings The effects of moisture on plant height, tillers, stem biomass, leaf biomass, leaf area, leaf mass, net photosynthetic rate, transpiration rate, water use efficiency were significant. The effects of fertilizers on tillers, stem biomass, net photosynthetic rate, transpiration rate, water use efficiency were significant. And the combination of fertilizers and moisture had a significant influence on tillers, stem biomass, transpiration rate and

收稿日期Received: 2019-02-25 接受日期Accepted: 2019-06-10

基金项目：国家自然科学基金(41771058)、国家重点研发计划(2016YFC0501200)、国家重点基础研究发展计划(2015CB150802)、科技部基础调查专 项(2015FY110500)、中国科学院A类战略性先导科技专项(XDA2306040303)。Supported by the National Natural Science Foundation of China (41771058), the National Key R\&D Program of China (2016YFC0501200), the National Basic Research Program of China (2015CB150802), the National Key Basic Survey of Resources (2015FY110500), and the Category A Strategic Leading Science and Technology Project of Chinese Academy of Sciences (XDA2306040303).

* 通信作者Corresponding author (mahongyuan@iga.ac.cn) 
water use efficiency (two-way ANOVA). The pattern of functional traits in response to precipitation differed between plants with varied fertilizer additions. Tillers and leaf area were increased in treatments with HW under $\mathrm{N}$ and $\mathrm{N}+\mathrm{P}$ additions, but not changed under $\mathrm{CK}$ and $\mathrm{P}$ addition. Stem biomass increased along the precipitation gradients under $\mathrm{CK}, \mathrm{N}$ addition and $\mathrm{P}$ addition, but did not change under $\mathrm{N}+\mathrm{P}$ additions. Specific leaf area with $\mathrm{HW}$ was significantly higher than that of MW under $\mathrm{N}$ addition, but not changed under other nutrient addition. Short-term nutrient additions significantly affected photosynthetic physiological traits of L. chinensis, but it had no significant effect on morphological traits under the same precipitation. The aboveground biomass of $L$. chinensis increased with the increase of precipitation, and reached the highest level of $522.55 \mathrm{~g} \cdot \mathrm{m}^{-2}$ with $\mathrm{HW}$ treatment under $\mathrm{N}$ addition. In conclusion, our results indicate that the functional traits in $L$. chinensis respond to precipitation addition and the patterns of responses differ under different nutrient additions, reflecting the adaptation to changes in water and nutrient availability.

Key words plant functional traits; morphological traits; physiological traits; aboveground biomass

Zhao DD, Ma HY, Li Y, Wei JP, Wang ZC (2019). Effects of water and nutrient additions on functional traits and aboveground biomass of Leymus chinensis. Chinese Journal of Plant Ecology, 43, 501-511. DOI: 10.17521/cjpe.2019.0041

植物功能性状是指植物具有的与其定植、存活、 生长和死亡紧密相关的一系列核心植物属性，且这 些属性能够反映植物个体对环境的响应和适应, 将 环境变化同植物群落的结构和功能联系起来(Díaz et al., 2007; Swenson \& Enquist, 2007; Violle et al., 2007; Lavorel et al., 2011; 刘晓娟和马克平, 2015)。 全球变化和植物功能性状之间的关系是当前植物生 态学研究的热点问题。

水分和养分是植物生产力水平提高的关键因素 (李生秀等, 1994; 徐学选等, 1995; 汪德水, 1999; 李开峰等, 2010), 二者相互作用、互相影响, 从而对 植物的生长发育以及产量产生水肥耦合效应(张广 涛等, 2007)。水分变化在植物的生长发育和生理生 态过程中扮演着重要角色, 当降水量增加时, 植物 能够通过调节其叶片干物质含量、比叶面积来适应 环境(赵新风等, 2014; Kunstler et al., 2016); 随着降 水量的增加, 植物的叶质量也会减小(Wright et al., 2004)。一般情况下, 植物地上生物量随降水量的增 加而增加(Hu et al., 2010; 张志南等, 2014)。虽然近 年来降水量与地上生物量之间的正相关关系已经逐 渐得到广泛证实, 但其关系形态在不同研究中还存 在一定差异(Yang et al., 2008; Zhang et al., 2019)。土 壤水分显著影响土壤养分有效性乃至陆地生态系统 养分循环进程, 合理控水对于提高生产力具有重要 意义。

氮 $(\mathrm{N})$ 和磷 $(\mathrm{P})$ 是植物生长的关键养分, 也是陆 地生态系统生产力的主要限制因素。尽管羊草 (Leymus chinensis)生长主要受N限制, 但在一定条 件下也受P限制(黄菊莹等, 2012; 白雪等, 2014)。黄
菊䒯等(2009)研究表明, 氮肥添加能够提高土壤氮 的有效性而对土壤磷的影响较小。因此, 在施氮肥 的同时, 需要适当增加土壤中 $\mathrm{P}$ 的含量, 以保持土 壤中植物可利用资源的平衡(Güsewell, 2004)。施加 $\mathrm{N} 、 \mathrm{P}$ 可能会改变土壤中其他养分的有效性，从而间 接影响土壤热量、微生物活性和土壤水分含量 (Behera \& Panda, 2009; Bobbink et al., 2010; 周鹏等, 2010; Lü et al., 2016), 养分添加能够影响植物的生 长和生物量分配(李德军等, 2004)、形态性状与生理 性状(万宏伟等, 2008; Lü et al., 2013), 进而影响群 落结构和组成, 使生态系统功能发生变化(Bai et al., 2010)。因此，水肥多因子交互作用对于植物生长发 育以及产量形成的影响对于探讨全球变化背景下植 物的响应有重要意义。

羊草属于禾本科赖草属(Leymus)多年生根茎禾 草, 克隆繁殖能力很强, 具有很广泛的生态适应性, 是我国北方典型草原的主要建群种或优势种(李永 宏, 1993; 易津等, 2001; 刘公社和李晓峰, 2011)。羊 草适应性强, 抗寒、抗旱、耐㾑薄、耐盐碱, 对于改 善我国草原生态环境意义重大(Ma et al., 2015)。然 而, 气候变化和近几十年人类过度开垦和放牧等活 动导致羊草草地退化 (祝廷成, 2004; Xu \& Zhou, 2006)。因此, 本研究通过设置降水量、氮肥和磷肥 的盆栽控制试验, 研究羊草主要功能性状和羊草生 物量对水分、养分的响应格局, 探讨水肥调控对羊 草功能性状及羊草生物量的影响, 旨为深入理解松 嫩草地重要优势物种羊草对降水变化和土壤养分变 化的响应机理。

www.plant-ecology.com 


\section{1 材料和方法}

\section{1 试验设计}

盆栽试验开始于2016年6月中旬, 在中国科学 院东北地理与农业生态研究所 $\left(125.38^{\circ} \mathrm{E}, 43.98^{\circ} \mathrm{N}\right.$, 海拔 $190 \mathrm{~m}$ )进行, 该区域气候属于温带季风气候, 1953-2012年的平均年降水量为 $498.0 \mathrm{~mm}$, 年降水 量极大值为 $754.0 \mathrm{~mm}$ (1956年), 比平均水平高出约 $50 \%$, 年降水量极小值为 $244.1 \mathrm{~mm}$ (1982年)(李晶等, $2015)$, 比平均水平低 $50 \%$ 。土壤类型为黑土, 有机 质平均含量为 $2.83 \%$ 、全 $\mathrm{N}$ 含量为 $1368.56 \mathrm{mg} \cdot \mathrm{kg}^{-1}$ 、 全P含量为 $669.18 \mathrm{mg} \cdot \mathrm{kg}^{-1}$ 。试验在遮雨棚内进行, 试验盆直径 $30 \mathrm{~cm}$, 高 $27 \mathrm{~cm}$, 盆内装入试验地附近 的0-20 cm 土层的过篮土, 每盆移栽 10 株大小均一 的羊草。晴天时收起遮雨棚, 下雨时进行遮挡以防 止盆栽接收自然降水。定期浇水, 剔除杂草, 保证羊 草植株生长。

2017年6月中旬至8月下旬对盆栽羊草进行水肥 调控。水肥调控试验包括 4 个施肥处理和 3 个降水处 理, 每个处理 3 个重复, 共36盆羊草, 研究不同水肥 处理以及水肥交互作用对羊草功能性状的影响。施 肥处理包括对照、单施氮肥、单施磷肥和氮磷共施 $(C K 、 N A 、 P A 、 N+P)$, 氮肥施用量为 $22.7 \mathrm{~g}$ 尿素 $\cdot \mathrm{m}^{-2}$, 磷肥施用量为 $200 \mathrm{~g}$ 过磷酸钙 $\cdot \mathrm{m}^{-2}$ 。水分处理包括 3 个降水量水平, 以多年平均降水量为对照, 在此基 础上增减水 $50 \%$, 分别用 MW (平均年降水量 $498 \mathrm{~mm}$ )、 $\mathrm{HW}$ (平均年降水量 $+50 \%, 747 \mathrm{~mm}$ )、 LW (平均年降水量 $-50 \%, 249 \mathrm{~mm}$ ) 表示, 灌溉用水为地 下水, 并且地下水中 $\mathrm{N}, \mathrm{P}$, 钾 $(\mathrm{K})$ 的浓度都在检测线 以下, 灌水频率为 2 天 1 次, 灌水量分别为: LW, 96 $\mathrm{mL} \cdot \operatorname{pot}^{-1} ; \mathrm{MW}, 193 \mathrm{~mL} \cdot \operatorname{pot}^{-1} ; \mathrm{HW}, 289 \mathrm{~mL} \cdot \operatorname{pot}^{-1}$ 。 8 月下旬进行指标测定, 并收割羊草。

\section{2 取样和测定方法}

\subsection{1 生长指标测定}

本试验生长指标主要包括植株高度、分等数、 茎生物量、叶生物量。测定羊草的植株高度和分蘖 数后, 每盆选取 20 株健康、叶片无损的植株放入塑 料袋中, 编号。用冰块敷于样株周围, 拿回实验室迅 速用剪刀、镊子等将羊草的茎与叶分开, 叶鞘包含 在叶片中, $80{ }^{\circ} \mathrm{C}$ 烘干 $24 \mathrm{~h}$ 至恒质量, 分别称质量。

\subsection{2 叶形态性状指标测定}

叶形态性状指标主要包括叶面积 $(L A)$ 、叶质量
$(L M)$ 、比叶面积 $(S L A)$ 。每盆选取 10 个最近完全展 开的健康叶片, 用叶面积仪(LI-3100, LI-COR, Lincoln, USA)测定叶面积。然后, $80{ }^{\circ} \mathrm{C}$ 烘干 $24 \mathrm{~h}$ 至恒质量, 称质量。比叶面积采用公式 $S L A=$ $L A / L M$ 计算。

\subsection{3 光合作用指标测定}

测定的光合作用指标包括净光合速率 $\left(P_{\mathrm{n}}\right)$ 、蒸 腾速率 $\left(T_{\mathrm{r}}\right)$ 、水分利用效率 $(W U E)$ 。净光合速率和蒸 腾速率的测定时间为8:00-11:30。每个处理选取3个 最近完全展开的健康全叶, 采用开放式气体交换系 统光合仪(LI-6400, LI-COR, Lincoln, USA)测定。测 定过程中, 叶室的 $\mathrm{CO}_{2}$ 浓度控制在 $400 \mu \mathrm{mol} \cdot \mathrm{mol}^{-1}$, 光强为 $1500 \mu \mathrm{mol} \cdot \mathrm{m}^{-1} \cdot \mathrm{s}^{-1}$ 。水分利用效率采用如下 公式计算: $W U E=P_{\mathrm{n}} / T_{\mathrm{r}}$ 。

\section{3 数据分析}

采用SPSS 20.0和Excel 2016对数据进行整理和 分析。采用双因素方差分析, 将不同水分添加水平 $(\mathrm{LW} 、 \mathrm{MW} 、 \mathrm{HW}) 、$ 养分添加水平 $(\mathrm{CK} 、 \mathrm{NA} 、 \mathrm{PA} 、$ $\mathrm{N}+\mathrm{P})$ 添加处理作为固定因子, 分析不同水肥添加水 平及水肥交互作用对羊草功能性状的影响。同一施 肥水平下, 不同水分添加处理之间的差异采用单因 素方差分析和邓肯多重比较法(Duncan' multiple range rest), 在显著性分析中, $p<0.05$ 为差异显著, $p>0.05$ 为差异不显著。数据均采用SPSS 20.0进行 统计分析, 统计图形均在Origin 8.5 中绘制。

\section{2 结果}

\section{1 羊草生长特性对水肥添加的响应}

羊草植株高度和叶生物量仅受到降水量的显著 影响 $(p<0.05)$, 施肥处理和水肥交互作用影响不显 著 $(p>0.05)$, 羊草分菜数和茎生物量受到水处理、 施肥处理和水肥交互作用显著影响 $(p<0.05)$ (表1)。 其中, 无养分添加和氮磷共施条件下，MW降水量 下羊草株高最高, 分别比 LW 降水量下提高了 $28.91 \%$ 和 $29.85 \%$; 单施氮肥和单施磷肥条件下，羊 草株高随着降水量的增加而升高。无养分添加和单 施磷肥条件下，降水量对羊草分藥数影响不显著; 单施氮肥和氮磷共施条件下, 分真数随降水量增加 而增加, 其中 HW 降水量下分藥数分别是 LW下的 2.42 倍和2.70倍(图1)。

图2是羊草茎叶生物量分配对水肥添加的响应, 
表1＼cjkstart水处理、施肥处理及其交互作用对羊草功能性状影响的双因素方差分析

Table 1 Results of two-way ANOVAs for the effects of water treatments, fertilization treatment and their interactions on functional traits of Leymus chinensis

\begin{tabular}{|c|c|c|c|c|c|c|c|c|c|c|}
\hline & \multirow[t]{2}{*}{ 因素 Factor } & \multirow[t]{2}{*}{ d.f. } & \multicolumn{2}{|c|}{$H$} & \multicolumn{2}{|c|}{$T$} & \multicolumn{2}{|c|}{$S B$} & \multicolumn{2}{|c|}{$L B$} \\
\hline & & & $F$ & $p$ & $F$ & $p$ & $F$ & $p$ & $F$ & $p$ \\
\hline \multirow{5}{*}{$\begin{array}{l}\text { 生长特性 } \\
\text { Growth } \\
\text { feature }\end{array}$} & 水分 Water (W) & 2 & 27.819 & $<0.001$ & 18.582 & $<0.001$ & 23.314 & $<0.001$ & 25.679 & $<0.001$ \\
\hline & 施肥 Fertilization (F) & 3 & 2.307 & $\mathrm{~ns}$ & 3.436 & $<0.05$ & 3.600 & $<0.05$ & 2.533 & ns \\
\hline & 水肥交互 $\mathrm{W} \times \mathrm{F}$ & 6 & 1.796 & $\mathrm{~ns}$ & 2.925 & $<0.05$ & 5.637 & $<0.01$ & 2.372 & ns \\
\hline & & & \multicolumn{2}{|c|}{$L A$} & \multicolumn{2}{|c|}{$L M$} & \multicolumn{2}{|c|}{ SLA } & & \\
\hline & & & $F$ & $p$ & $F$ & $p$ & $F$ & $p$ & & \\
\hline \multirow{5}{*}{$\begin{array}{l}\text { 形态性状 } \\
\text { Morphological } \\
\text { trait }\end{array}$} & 水分 $\mathrm{W}$ & 2 & 10.384 & $<0.01$ & 6.842 & $<0.01$ & 0.846 & $\mathrm{~ns}$ & & \\
\hline & 施肥 $\mathrm{F}$ & 3 & 1.416 & ns & 1.422 & $\mathrm{~ns}$ & 2.991 & $\mathrm{~ns}$ & & \\
\hline & 水肥交互 $\mathrm{W} \times \mathrm{F}$ & 6 & 0.610 & $\mathrm{~ns}$ & 1.424 & $\mathrm{~ns}$ & 1.528 & ns & & \\
\hline & & & \multicolumn{2}{|c|}{$P_{\mathrm{n}}$} & \multicolumn{2}{|c|}{$T_{\mathrm{r}}$} & \multicolumn{2}{|c|}{ WUE } & & \\
\hline & & & $F$ & $p$ & $F$ & $p$ & $F$ & $p$ & & \\
\hline \multirow{3}{*}{$\begin{array}{l}\text { 生理性状 } \\
\text { Physiological } \\
\text { trait }\end{array}$} & 水分 $\mathrm{W}$ & 2 & 115.990 & $<0.001$ & 86.229 & $<0.001$ & 105.150 & $<0.001$ & & \\
\hline & 施肥 $F$ & 3 & 6.948 & $<0.001$ & 5.325 & $<0.01$ & 3.972 & $<0.05$ & & \\
\hline & 水肥交互 $\mathrm{W} \times \mathrm{F}$ & 6 & 1.750 & ns & 5.608 & $<0.001$ & 4.828 & $<0.001$ & & \\
\hline
\end{tabular}

显著性差异 $(p<0.05)$ 用粗体显示, $n$ s表示无显著性差异 $(p>0.05)$ 。 $H$, 株高; $L A$, 叶面积; $L B$, 叶生物量; $L M$, 叶质量; $P_{\mathrm{n}}$, 净光合速率; $S B$, 茎生物量; $S L A$, 比叶面积; $T$, 分蔝数; $T_{\mathrm{r}}$, 蒸腾速率; WUE, 水分利用效率。

Significant differences $(p<0.05)$ are highlighted in bold; ns means non-significant $(p>0.05)$. $H$, height; $L A$, leaf area; $L B$, leaf biomass; $L M$, leaf dry mass; $P_{\mathrm{n}}$, net photosynthetic rate; $S B$, stem biomass; $S L A$, specific leaf area; $T$, tillers; $T_{\mathrm{r}}$, transpiration rate; $W U E$, water use efficiency.

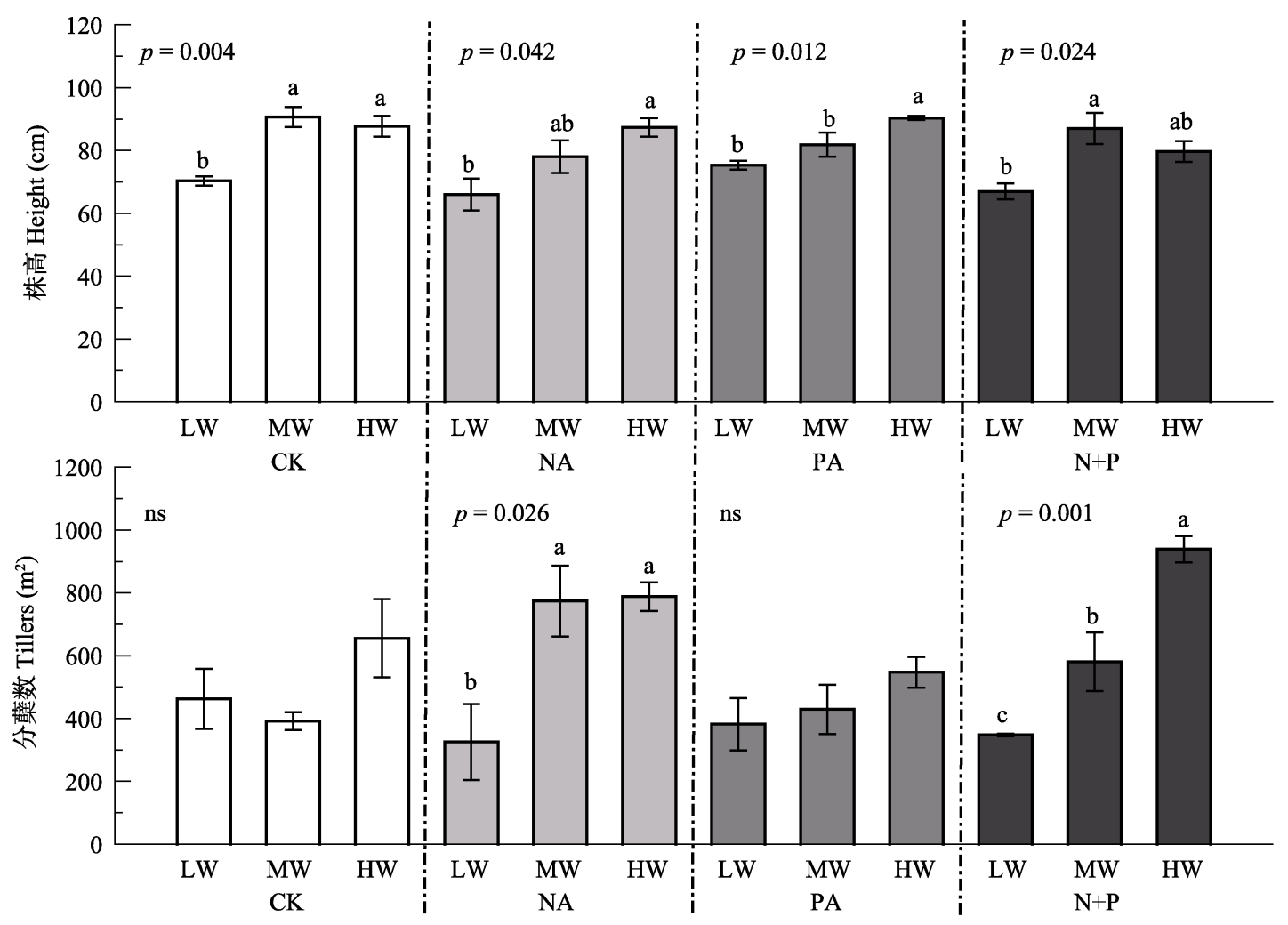

处理 Treatment

图1 水肥添加对羊草株高和分蒕的影响(平均值土标准误差)。CK, 对照; NA, 氮添加; PA, 磷添加; N+P, 氮、磷共同添加; LW、 MW、HW分别表示低、中、高降水量水平。不同小写字母表示在相同施肥水平下，不同水分添加处理间差异显著 $(p<0.05)$; ns 表示水分处理间差异不显著 $(p>0.05)$ 。

Fig. 1 Effects of fertilization and water on height and tillers of Leymus chinensis (mean $\pm S E$ ). CK, control; NA, N addition; PA, P addition; N+P, N and P additions; LW, MW, HW represent low, moderate and high precipitation levels. Different lowercase letters indicate significant difference $(p<0.05)$ among different water treatments at the same fertilizer addition, and ns indicates non-significant differences $(p>0.05)$ among water treatments.

www.plant-ecology.com 


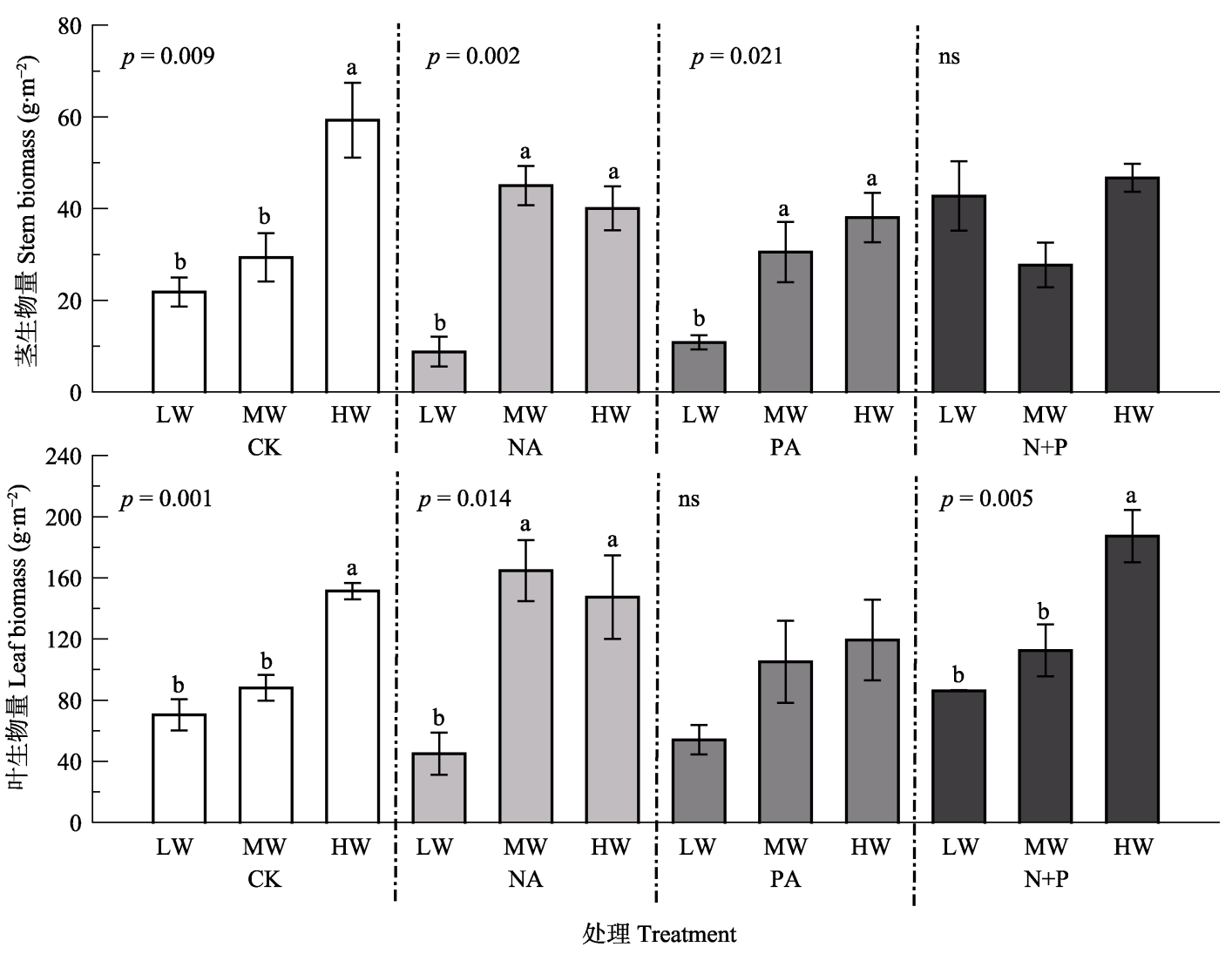

图2 水肥添加对羊草茎叶生物量分配的影响(平均值土标准误差)。CK, 对照; NA, 氮添加; PA, 磷添加; N+P, 氮、磷共同添加; LW、MW、HW分别表示低、中、高降水量水平。不同小写字母表示在相同施肥水平下，不同水分添加处理间差异显著 $(p<0.05)$; $\mathrm{ns}$ 表示水分处理间差异不显著 $(p>0.05)$ 。

Fig. 2 Effects of fertilization and water on biomass allocation of Leymus chinensis (mean $\pm S E$ ). CK, control; NA, N addition; PA, P addition; N+P, N and P additions; LW, MW, HW represent low, moderate and high precipitation levels. Different lowercase letters indicate significant difference $(p<0.05)$ among different water treatments at the same fertilizer addition, and ns indicates non-significant differences $(p>0.05)$ among water treatments.

无养分添加和单施磷肥条件下，羊草茎生物量随着 降水量增加而增加; 氮磷共施条件下，不同降水量 对羊草茎生物量没有显著影响; 单施氮肥条件下, MW降水量下茎生物量最高, 是LW下的5.12倍。无 养分添加和氮磷共施条件下, 羊草叶生物量随着降 水量的增加而增加; 单施磷肥条件下，羊草叶生物 量随降水量增加而增加, 但是差异不显著; 单施氮 肥条件下, MW降水量下叶生物量最高, 是LW下的 3.67倍。

\section{2 羊草叶形态性状对水肥添加的响应}

降水量显著影响了羊草叶面积和叶质量 $(p<$ $0.05)$, 仅对比叶面积无显著影响 $(p>0.05)$, 而施肥 处理和水肥交互作用对羊草叶形态性状均无显著影 响 $(p>0.05)$ (表1)。其中, 相同施肥处理下, 羊草叶 面积整体表现为随降水量增加而增加。单施氮肥时, HW 条件下羊草的比叶面积最低, 分别比LW和MW 低了 $17.55 \%$ 和 $20.99 \%$ (图3)。

\section{3 羊草光合生理性状对水肥添加的响应}

如表1所示，降水量和施肥处理对羊草净光合 速率、蒸腾速率和水分利用效率均有显著影响 $(p<$ $0.05)$, 水肥交互作用对羊草蒸滕速率和水分利用效 率有显著影响 $(p<0.05)$, 因此, 羊草的光合生理性 状对降水量和养分添加的响应非常明显。单施氮肥 和单施磷肥条件下, 羊草净光合速率随降水量增加 而增加，而不添加养分和氮磷共施条件下，MW降 水量下羊草净光合速率最高; 整体上羊草水分利用 效率随着降水量的增加而增加(图4)。

\section{4 羊草地上生物量对水肥添加的响应}

羊草地上生物量受到降水量和施肥处理的显著 影响。如图5所示, 4 种施肥处理下, 羊草地上生物量随 着降水量的增加显著升高 $(p<0.05)$, 无养分添加条件 下, MW、HW降水量下羊草地上生物量分别是 LW条 件下生物量的1.62倍和 2.52 倍。单施氮肥条件下, HW 降水量使得羊草生物量达到最高, 为 $522.55 \mathrm{~g} \cdot \mathrm{m}^{-2}$ 。 

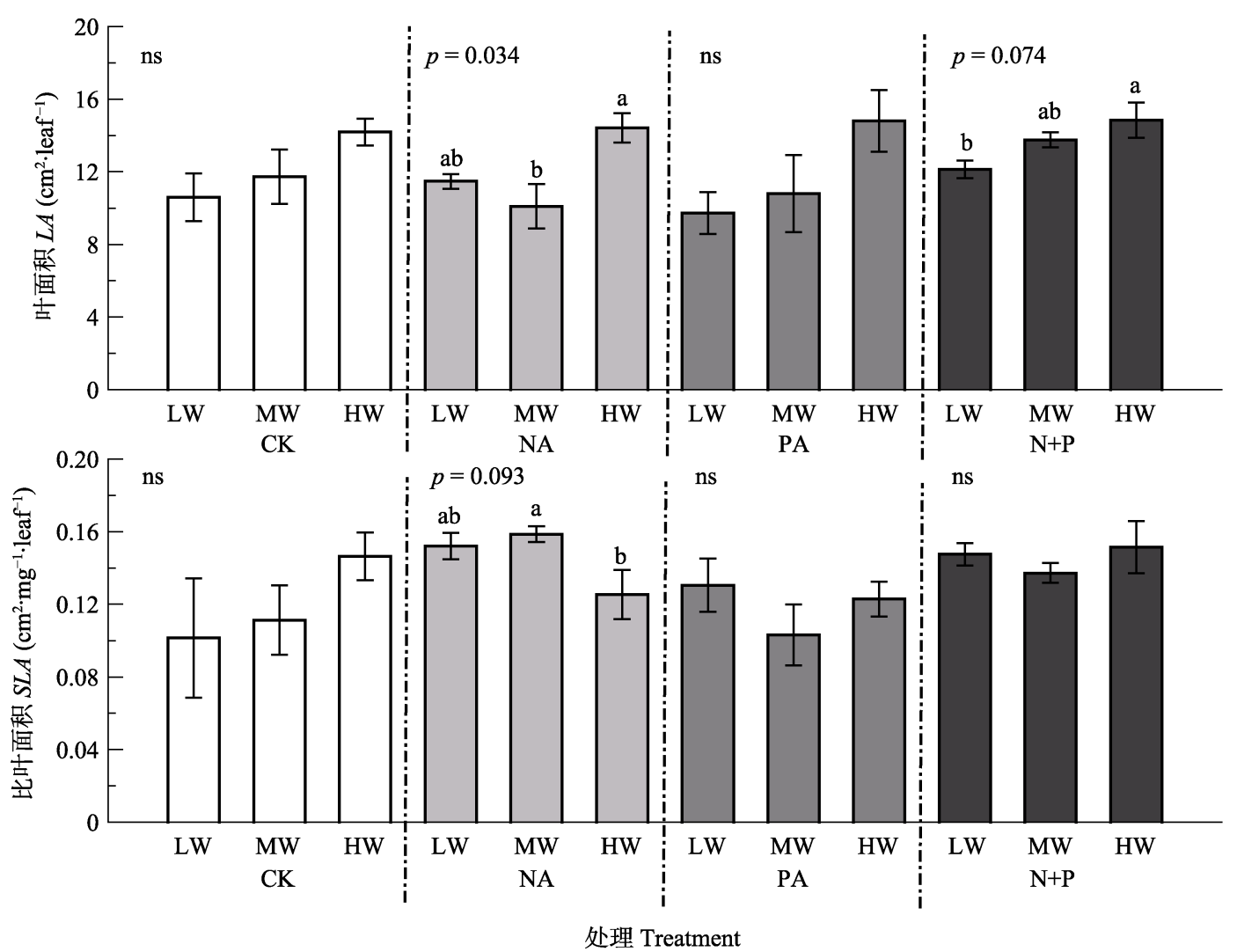

图3 水肥添加对羊草叶片形态性状的影响(平均值土标准误差)。CK, 对照; NA, 氮添加; PA, 磷添加; N+P, 氮、磷共同添加; LW、MW、HW分别表示低、中、高降水量水平。不同小写字母表示在相同施肥水平下，不同水分添加处理间差异显著 $(p<0.05)$; $n s$ 表示水分处理间差异不显著 $(p>0.05)$ 。

Fig. 3 Effects of fertilization and water on morphological traits of Leymus chinensis (mean $\pm S E$ ). LA, leaf area; SLA, specific leaf area. CK, control; NA, N addition; PA, P addition; N+P, N and P additions; LW, MW, HW represent low, moderate and high precipitation levels. Different lowercase letters indicate significant difference $(p<0.05)$ among different water treatments at the same fertilizer addition, and ns indicates non-significant differences $(p>0.05)$ among water treatments.

\section{3 讨论}

\section{1 水分和养分添加对羊草功能性状的影响}

功能性状作为解释变量, 对生态系统功能和服 务的变化有显著的指示作用(Garnier et al., 2004; Mokany et al., 2010; Moor et al., 2015; Osborne et al., 2018)，能够显示植物对环境异质性的适应能力 (Reich \& Oleksyn, 2004; Faucon et al., 2017)。植被生 长过程中, 功能性状会受到降水量和施肥处理不同 程度的影响。降水量增加使植株高度增高, 叶面积 增大, 净光合速率升高(曾小平等, 2004; 许振柱和 周广胜, 2005; 孟凡超等, 2014; Yue et al., 2019), 本 研究结果与之一致, 主要原因是水分充足与否直接 影响到羊草根系的发育, 根系发育的良好程度又直 接影响到植物对土壤养分的吸收及养分在植物体内 的循环, 进而对植物功能性状产生影响(李秀芳, 2011; Balachowski \& Volaire, 2018)。已有研究表明,
植物的光合能力与相对生长速率紧密相关(Shipley, 2006; Morgan et al., 2011), 叶面积反映了叶片对光 的截获能力(Poorter \& Rozendaal, 2008)。因此，降水 量增加与氮磷共施条件下较高的光合速率和较大的 叶面积可能是较高的叶生物量和茎生物量的主要原 因。Fonseca等(2000)的研究表明澳大利亚东南部多 年生植物的SLA随降水量的增加而增加; 驼线藜 (Ceratoides latens)的SLA与降水量呈正相关关系(朱 军涛等, 2010); 植物叶质量也会随降水量的增加而 减小(Wright et al., 2004; Rosbakh et al., 2015; Buckley et al., 2019)。而本研究中, 降水量对羊草SLA没 有显著影响 $(p>0.05)$, 可能是由于羊草对短期水分 处理响应不明显(Gao et al., 2015; Forrestel et al., 2017)。

短期的养分添加主要影响羊草的生理性状, 如 叶片光合速率、蒸腾速率和水分利用效率均显著高 于不施肥处理，这些性状主要与光合作用相关。这 


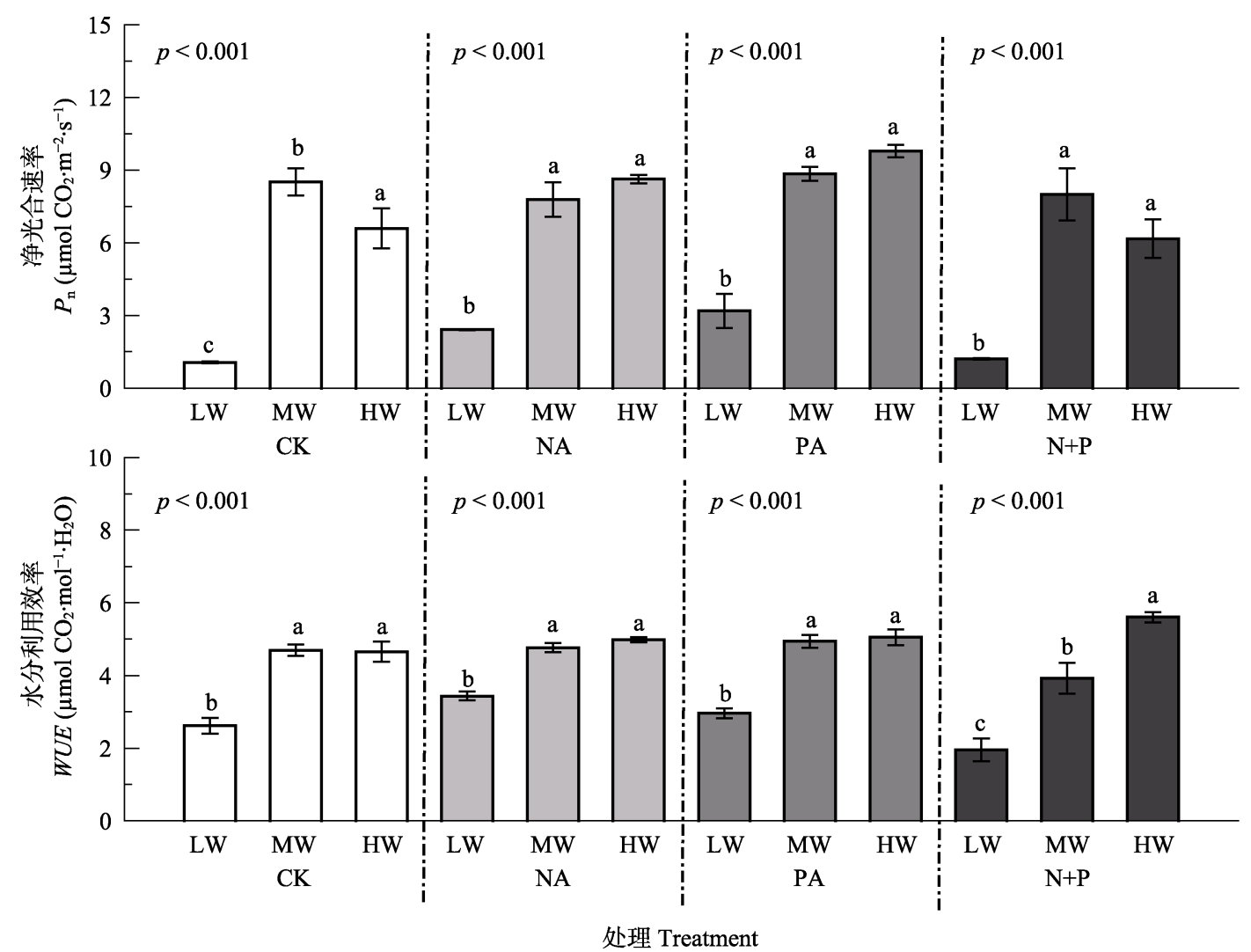

图4 水肥添加对羊草叶片生理性状的影响(平均值土标准误差)。CK, 对照; NA, 氮添加; PA, 磷添加; N+P, 氮、磷共同添加; LW、MW、HW分别表示低、中、高降水量水平。不同小写字母表示在相同施肥水平下，不同水分添加处理间差异显著 $(p<0.05)$; $n s$ 表示水分处理间差异不显著 $(p>0.05)$ 。

Fig. 4 Effects of fertilization and water on photosynthetic traits of Leymus chinensis (mean $\pm S E$ ). $P_{\mathrm{n}}$, net photosynthesis rate; WUE, water use efficiency. CK, control; NA, N addition; PA, P addition; N+P, N and P additions; LW, MW, HW represent low, moderate and high precipitation levels. Different lowercase letters indicate significant difference $(p<0.05)$ among different water treatments at the same fertilizer addition, and ns indicates non-significant differences $(p>0.05)$ among water treatments.

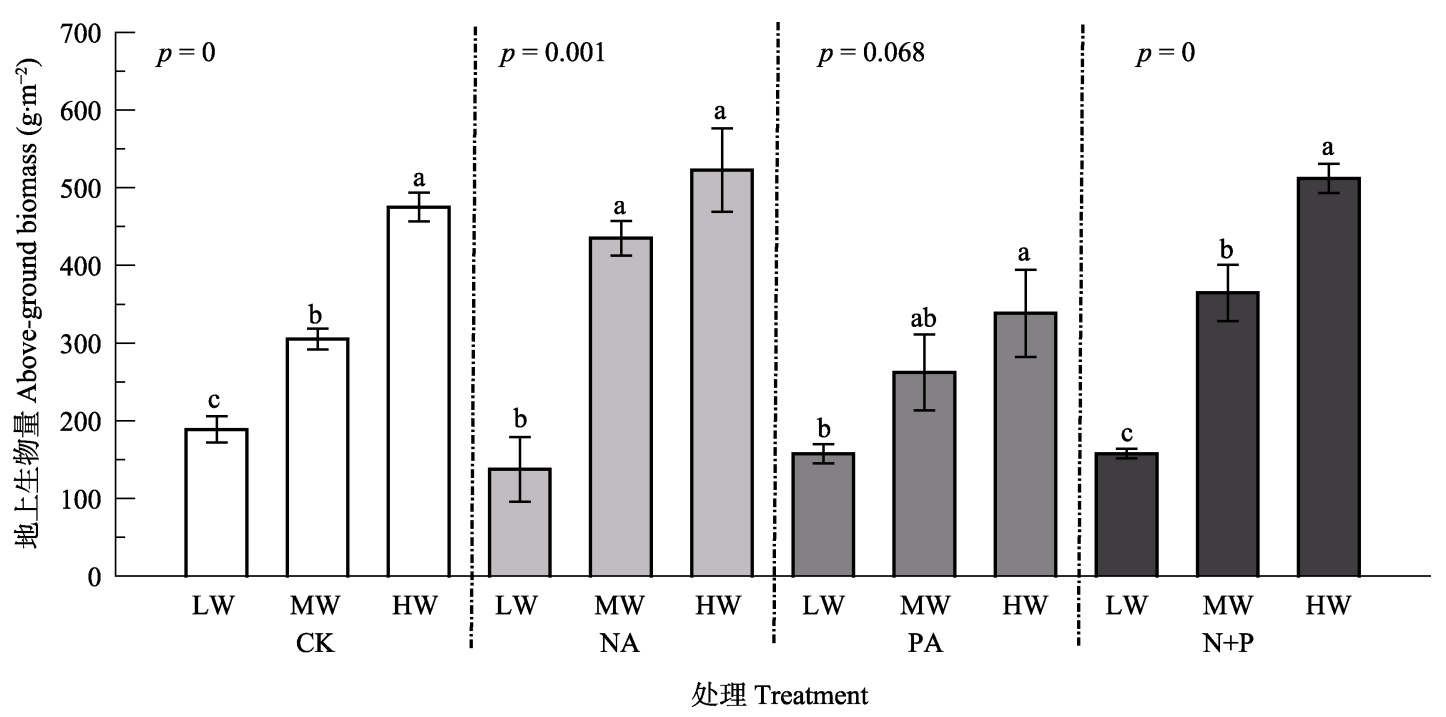

图5 水肥添加对羊草地上生物量的影响(平均值土标准误差)。CK, 对照; NA, 氮添加; PA, 磷添加; N+P, 氮、磷共同添加; LW、 MW、HW分别表示低、中、高降水量水平。不同小写字母表示在相同施肥水平下，不同水分添加处理间差异显著 $(p<0.05) ; \mathrm{ns}$ 表示水分处理间差异不显著 $(p>0.05)$ 。

Fig. 5 Effects of fertilization and water on above-ground biomass of Leymus chinensis (mean $\pm S E$ ). CK, control; NA, N addition; PA, P addition; N+P, N and P additions; LW, MW, HW represent low, moderate and high precipitation levels. Different lowercase letters indicate significant difference $(p<0.05)$ among different water treatments at the same fertilizer addition, and ns indicates non-significant differences $(p>0.05)$ among water treatments. 
说明养分添加缓解了植物对养分的竞争, 使物种对 资源的竞争从地下部分转向地上部分, 即增加植物 对光资源的竞争(Bobbink et al., 2010)。然而, 养分 添加对羊草形态性状的影响作用相对较小, 这与万 宏伟等(2008)的研究结果不一致, 可能是因为本研 究时间较短, 植物性状随着资源水平的改变, 首先 是调整生理性状使其快速适应，而形态性状相对滞 后, 需要较长的时间才能发生显著变化(宋彦涛等, 2016; Yang et al., 2019)。

\section{2 水分和养分添加对羊草地上生物量的影响}

大部分陆地生态系统都受到 $\mathrm{N}$ 和P限制，适当 添加养分可以提高植物生产力(LeBauer \& Treseder, 2008; Djaman et al., 2018)。有学者指出氮供应充分 可以提高植物的光合能力, 进而促进植物的生长, 提高植物生物量(Chen et al., 2005; Jan et al., 2018)。 在干旱半干旱地区, 植物生长不仅受到养分的限制, 较低的降水量导致水分也成为该地区的主要限制因 子(Rao \& Allen, 2010)。本研究结果表明, 降水量增 加显著提高了羊草地上生物量。在减少降水量的情 况下，氮添加、氮磷共同添加对羊草地上生物量的 促进效应明显低于正常和增加降水量。这是因为植 物对养分的吸收和运输往往依赖于水分, 养分要溶 解于水中才能被植物吸收, 同时植物地上部分的蒸 滕作用拉力成为养分运输的动力 (Barker et al., 2005)。另外土壤中氮的矿化和硝化作用与水分也有 密切的联系(王双, 2008)。因此, 干旱可能使溶解于 水中的养分降低, 同时使植物的蒸腾作用降低, 导 致减少降水时养分添加对植物的促进作用弱于其他 处理。

综上所述，羊草功能性状在不同水分和养分添 加下的响应策略不同，水肥交互作用对羊草的多种 功能性状影响显著, 羊草株高、分藥、叶面积、光 合作用及地上生物量随降水量的增加而升高, 短期 氮磷处理显著影响羊草叶片光合生理性状, 而对羊 草叶形态性状没有显著影响。适当的水肥组合能够 提高羊草地上生物量, 单施氮肥时, $747 \mathrm{~mm}$ 降水量 使得羊草生物量达到最高, 为 $522.55 \mathrm{~g} \cdot \mathrm{m}^{-2}$ 。因此, 在羊草草地的研究中, 应该注重发挥水肥耦合作 用。在未来降水格局发生变化, 大气氮沉降日益加 剧的情况下, 羊草功能性状的变化对草原生态系统 功能和服务的影响有待进一步研究。

\section{参考文献}

Bai X, Cheng JH, Zheng SX, Zhan SX, Bai YF (2014). Ecophysiological responses of Leymus chinensis to nitrogen and phosphorus additions in a typical steppe. Chinese Journal of Plant Ecology, 38, 103-115. [白雪, 程军回, 郑淑霞，詹书侠，白永飞 (2014). 典型草原建群种羊草 对氮磷添加的生理生态响应。植物生态学报，38, 103-115.]

Bai YF, Wu JG, Clark CM, Naeem S, Pan QM, Huang JH, Zhang LX, Han XG (2010). Tradeoffs and thresholds in the effects of nitrogen addition on biodiversity and ecosystem functioning: Evidence from Inner Mongolia Grasslands. Global Change Biology, 16, 358-372.

Balachowski JA, Volaire FA (2018). Implications of plant functional traits and drought survival strategies for ecological restoration. Journal of Applied Ecology, 55, 631-640.

Barker DH, Vanier C, Namburg E, Charlet TN, Nielsen KM, Newingham BA, Smith SD (2005). Enhanced monsoon precipitation and nitrogen deposition affect leaf traits and photosynthesis differently in spring and summer in the desert shrub Larrea tridentate. New Phytologist, 169, 799-808.

Behera SK, Panda RK (2009). Integrated management of irrigation water and fertilizers for wheat crop using field experiments and simulation modeling. Agricultural Water Management, 96, 1532-1540.

Bobbink R, Hicks K, Galloway J, Spranger T, Alkemade R, Ashmore M, Bustamante M, Cinderby S, Davidson E, Dentener F, Emmett B, Erisman JW, Fenn M, Gilliam F, Nordin A, Pardo L, de Vries W (2010). Global assessment of nitrogen deposition effects on terrestrial plant diversity: A synthesis. Ecological Applications, 20, 30-59.

Buckley H, Young CA, Charlton ND, Hendricks WQ, Haley B, Nagabhyru P, Rudgers JA (2019). Leaf endophytes mediate fertilizer effects on plant yield and traits in northern oat grass (Trisetum spicatum). Plant and Soil, 434, 425-440.

Chen SP, Bai YF, Zhang LX, Han XG (2005). Comparing physiological responses of two dominant grass species to nitrogen addition in Xilin River Basin of China. Environmental and Experimental Botany, 53, 65-75.

Díaz S, Lavorel S, Mcintyre S, Falczuk V, Casanoves F, Milchunas DG, Skarpe C, Rusch G, Sternberg M, Noy-Meir I, Landsberg J, Zhang W, Clark H, Campbell BD (2007). Plant trait responses to grazing-A global synthesis. Global Change Biology, 13, 313-341.

Djaman K, Mel VC, Diop L, Sow A, EI-Namaky R, Manneh B, Satio K, Futakuchi K, Irmak S (2018). Effects of alternate wetting and drying irrigation regime and nitrogen fertilizer on yield and nitrogen use efficiency of irrigated rice in the Sahel. Water, 10, 711. DOI: 10.3390/w10060711. 
Faucon MP, Houben D, Lambers H (2017). Plant functional traits: Soil and ecosystem services. Trends in Plant Science, 22, 385-394.

Fonseca CR, Overton JM, Collins B, Westoby M (2000). Shifts in trait-combinations along rainfall and phosphorus gradients. Journal of Ecology, 88, 964-977.

Forrestel EJ, Donoghue MJ, Edwards EJ, Jetz W, du Toit JCO, Smith MD (2017). Different clades and traits yield similar grassland functional responses. Proceedings of the $\mathrm{Na}$ tional Academy of Sciences of the United States of America, 114, 705-710.

Gao R, Yang X, Liu G, Huang Z, Walck JL (2015). Effects of rainfall pattern on the growth and fecundity of a dominant dune annual in a semi-arid ecosystem. Plant and Soil, 389, 335-347.

Garnier E, Cortez J, Billès G, Navas ML, Roumet C, Debussche M, Laurent G, Blanchard A, Aubry D, Bellmann A, Neil C, Toussaint JP (2004). Plant functional markers capture ecosystem properties during secondary succession. Ecology, 85, 2630-2637.

Güsewell S (2004). N:P ratios in terrestrial plants: Variation and functional significance. New Phytologist, 164, 243-266.

Hu ZM, Yu GR, Fan JW, Zhong HP, Wang SQ, Li SG (2010). Precipitation-use efficiency along a 4500-km grassland transect. Global Ecology and Biogeography, 19, 842-851.

Huang JY, Xu P, Yu HL, Yuan ZY, Li LH (2012). Responses of biomass, nutrient allocation of Leymus chinensis along N, P and water gradients. Pratacultural Science, 29, 1589-1595. [黄菊芗, 徐鹏, 余海龙, 袁志友, 李凌浩 (2012). 羊草生物量和养分分配对养分和水分添加的响 应. 草业科学, 29, 1589-1595.]

Huang JY, Yuan ZY, Li LH (2009). Changes in [N], [P] and specific leaf area of green leaves of Leymus chinensis along nitrogen, phosphorus and water gradients. Chinese Journal of Plant Ecology, 33, 442-448. [黄菊荣, 袁志友, 李凌浩 (2009). 羊草绿叶氮、磷浓度和比叶面积沿氮、 磷和水分梯度的变化. 植物生态学报, 33, 442-448.]

Jan R, Aga FA, Bahar FA, Singh T, Lone R (2018). Effect of nitrogen and silicon on growth and yield attributes of transplanted rice (Oryza sativa L.) under Kashmir conditions. Journal of Pharmacognosy and Phytochemistry, 7, 328-332.

Kunstler G, Falster D, Coomes DA, Hui F, Kooyman RM, Laughlin DC, Poorter L, Vanderwel M, Vieilledent G, Wright SJ, Aiba M, Baraloto C, Caspersen J, Cornrlissen JHC, Gourlet-Fleury S, Hanewinkel M, Herault B, Kattge J, Kurokawa H, Onoda Y, Peñuelas J, Poorter H, Uriarte M, Richardson S, Ruiz-Benito P, Sun IF, Ståhl G, Swenson NG, Thompson J, Westerlund B, Wirth C, Zavala MA, Zeng HC, Zimmermann JK, Zimmermann NE, Westoby M (2016). Plant functional traits have globally consistent effects on competition. Nature, 529, 204-207.

Lavorel S, Grigulis K, Lamarque P (2011). Using plant functional traits to understand the landscape distribution of multiple ecosystem services. Journal of Ecology, 99, 135-147.

LeBauer DS, Treseder KK (2008). Nitrogen limitation of net primary productivity in terrestrial ecosystems is globally distributed. Ecology, 89, 371-379.

Li DJ, Mo JM, Fang YT, Cai XA, Xue JH, Xu GL (2004). Effects of simulated nitrogen deposition on growth and photosynthesis of Schima superba, Castanopsis chinensis and Cryptocarya concinna seedlings. Acta Ecologica Sinica, 2004, 24，876-882. [李德军, 莫江明, 方运霆, 蔡锡安, 薛璟花, 徐国良 (2004). 模拟氮沉降对三种南亚热带树 苗生长和光合作用的影响. 生态学报, 24, 876-882.]

Li J, Liu ZS, Xue XQ, Yu XG, Zhang B, Yue YT (2015). Study of rainfall characteristics and pattern in Changchun city. China Water \& Wastewater, 31, 100-104. [李晶, 刘志生, 薛喜权, 于相国，张波，岳奕肜 (2015). 长春市降雨特 征及雨型分析研究. 中国给水排水, 31, 100-104.]

Li KF, Zhang FC, Qi YL, Xing YY, Li ZJ (2010). Effects of water-fertilizer spatial coupling in root zone on winter wheat growth and yield. Chinese Journal of Applied Ecology, 21，3154-3160. [李开峰, 张富仓, 祁有玲, 邢 英英, 李志军 (2010). 根区水肥空间耦合对冬小麦生长 及产量的影响. 应用生态学报, 21, 3154-3160.]

Li SX, Li SQ, Gao YJ, Wang XQ, He HX, Du JJ (1994). The mechanism and effects of $\mathrm{N}$ fertilization in increasing water use efficiency. Agricultural Research in the Arid Areas, 12, 38-46. [李生秀, 李世清, 高亚军, 王喜庆, 贺海香, 杜建军 (1994). 施用氮肥对提高旱地作物利用土壤水 分的作用机理和效果. 干旱地区农业研究, 12, 38-46.]

Li XF (2011). Effects of Water and Fertilizer Cooperation on the Growth Nutrient Absorption and Root Activity of Summer Maize. Master degree dissertation, Hebei Agricultural University, Baoding, Hebei. [李秀芳 (2011). 水 肥互作对夏玉米生长与养分吸收及根系活性的影响. 硕士学位论文, 河北农业大学, 河北保定.]

Li YH (1993). Grazing dynamics of the species diversity in Aneurolepidium chinense steppe and Stipa grandis steppe. Acta Botanica Sinica, 35, 877-884. [李永宏 (1993). 放 牧影响下羊草草原和大针茅草原植物多样性的变化. 植物学报, 35, 877-884.]

Liu GS, Li XF (2011). Study on Germplasm Resources of Leymus chinensis. Science Press, Beijing. [刘公社, 李晓峰 (2011). 羊草种质资源研究. 科学出版社, 北京.]

Liu XJ, Ma KP (2015). Plant functional traits-concepts, applications and future directions. Scientia Sinica Vitae, 45, 325-339. [刘晓娟, 马克平 (2015). 植物功能性状研究 进展. 中国科学: 生命科学, 45, 325-339.]

Lü XT, Reed S, Yu Q, He NP, Wang ZW, Han XG (2013). Convergent responses of nitrogen and phosphorus resorption 
to nitrogen inputs in a semiarid grassland. Global Change Biology, 19, 2775-2784.

Lü XT, Reed SC, Yu Q, Han XG (2016). Nutrient resorption helps drive intra-specific coupling of foliar nitrogen and phosphorus under nutrient-enriched conditions. Plant and Soil, 398, 111-120.

Ma HY, Yang HY, Liang ZW, Ooi MKJ (2015). Effects of 10 -year management regimes on the soil seed bank in salinealkaline grassland. PLOS ONE, 10, e0122319. DOI: 10.1371/ journal.pone.0122319.

Meng FC, Zhang JH, Yao FM (2014). Interactive effects of elevated $\mathrm{CO}_{2}$ concentration and increasing precipitation on yield and growth development in maize. Chinese Journal of Plant Ecology, 38, 1064-1073. [孟凡超, 张佳华, 姚风 梅 (2014). $\mathrm{CO}_{2}$ 浓度升高和降水增加协同作用对玉米产 量及生长发育的影响. 植物生态学报, 38, 1064-1073.]

Mokany K, Ash J, Roxburgh S (2010). Functional identity is more important than diversity in influencing ecosystem processes in a temperate native grassland. Journal of Ecology, 96, 884-893.

Moor H, Hylander K, Norberg J (2015). Predicting climate change effects on wetland ecosystem services using species distribution modeling and plant functional traits. Ambio, 44, 113-126.

Morgan JA, LeCain DR, Pendall E, Blumenthal DM, Kimball BA, Carrillo Y, Williams DG, Heisler-White J, Dijkstra FA, West $\mathrm{M}$ (2011). $\mathrm{C}_{4}$ grasses prosper as carbon dioxide eliminates desiccation in warmed semi-arid grassland. Nature, 476, 202-205.

Osborne CP, Charles-Dominique T, Stevens N, Bond WJ, Midgley G, Lehmann CER (2018). Human impacts in African savannas are mediated by plant functional traits. New Phytologist, 220, 10-24.

Poorter L, Rozendaal DMA (2008). Leaf size and leaf display of thirty-eight tropical tree species. Oecologia, 158, 35-46.

Rao LE, Allen EB (2010). Combined effects of precipitation and nitrogen deposition on native and invasive winter annual production in California deserts. Oecologia, 162, 1035-1046.

Reich PB, Oleksyn J (2004). Global patterns of plant leaf N and $\mathrm{P}$ in relation to temperature and latitude. Proceedings of the National Academy of Sciences of the United States of America, 101, 11001-11006.

Rosbakh S, Römermann C, Poschlod P (2015). Specific leaf area correlates with temperature: New evidence of trait variation at the population, species and community levels. Alpine Botany, 125, 79-86.

Shipley B (2006). Net assimilation rate, specific leaf area and leaf mass ratio: Which is most closely correlated with relative growth rate? A meta-analysis. Functional Ecology, 20, 565-574.

Song YT, Li Q, Wang P, Zhou DW, Wuyunna (2016). Re- sponse of Leymus chinensis functional traits and aboveground biomass to nitrogen addition in Songnen grassland in northeast China. Pratacultural Science, 33, 1383-1390. [宋彦涛, 李强, 王平, 周道玮, 乌云娜 (2016). 羊草功 能性状和地上生物量对氮素添加的响应. 草业科学, 33, 1383-1390.]

Swenson NG, Enquist BJ (2007). Ecological and evolutionary determinants of a key plant functional trait: Wood density and its community-wide variation across latitude and elevation. American Journal of Botany, 94, 451-459.

Violle C, Navas ML, Vile D, Kazakou E, Fortunerl C, Hummel I, Garnier E (2007). Let the concept of trait be functional! Oikos, 116, 882-892.

Wan HW, Yang Y, Bai SQ, Xu YH, Bai YF (2008). Variations in leaf functional traits of six species along a nitrogen addition gradient in Leymus chinensis steppe in Inner Mongolia. Journal of Plant Ecology (Chinese Version), 32, 611-621. [万宏伟, 杨阳, 白世勤, 徐云虎, 白永飞 (2008). 羊草草原群落6种植物叶片功能特性对氮素添 加的响应. 植物生态学报, 32, 611-621.]

Wang DS (1999). Synergistic Effect and Coupling Model of Fertilizer and Water in Dryland Farmland. China Meteorological Press, Beijing. [汪徳水 (1999). 旱地农田肥水 协同效应与耦合模式. 气象出版社, 北京.]

Wang S (2008). Effect of N Rates on Growth of Summer Maize and Drought Threshold under Different Drought Levels. Master degree dissertation, Huazhong Agricultural University, Wuhan. [王双 (2008), 干旱条件下施氮水平对 夏玉米生长及干旱阈值的影响. 硕士学位论文, 华中农 业大学, 武汉.]

Wright IJ, Reich PB, Westoby M, Ackerly DD, Baruch Z, Bongers F, Cavender-Bares J, Chapin T, Cornelissen JHC, Diemer M, Flexas J, Garnier E, Groom PK, Gulias J, Hikosaka K, Lamont BB, lee T, Lee W, Lusk C, Midgley JJ, Navas ML, Niinemets Ü, Oleksyn J, Dsada N, Poorter H, Poot P, Prior L, Pyankov VI, Roumet G, Thomas SC, Tjoelker MG, Veneklaas EJ, Villar R (2004). The worldwide leaf economics spectrum. Nature, 428, 821-827.

Xu XX, Chen GL, Mu XM (1995). The coordinative effect of moisture and fertilizer upon spring wheat yields. Agricultural Research in the Arid Areas, 13, 34-38. [徐学选，陈 国良，穆兴民 (1995). 水肥对春小麦产量的效应研究. 干旱地区农业研究, 13, 34-38.]

Xu ZZ, Zhou GS (2005). Effects of soil moisture on growth characteristics of Leymus chinensis seedlings under different temperature conditions. Chinese Journal of Ecology, 24, 256-260. [许振柱, 周广胜 (2005). 不同温度条件下 土壤水分对羊草幼苗生长特性的影响. 生态学杂志, 24 , 256-260.]

Xu ZZ, Zhou GS (2006). Nitrogen metabolism and photosynthesis in Leymus chinensis in response to long-term soil drought. Journal of Plant Growth Regulation, 25,

www.plant-ecology.com 
252-266.

Yang X, Li J, Zhao T, Mo L, Zhang J, Ren H, Zhao N, Gao Y (2019). Variation and heritability of morphological and physiological traits among Leymus chinensis genotypes under different environmental conditions. Journal of Arid Land, 11, 66-74.

Yang Y, Fang J, Ma W, Wang W (2008). Relationship between variability in aboveground net primary production and precipitation in global grasslands. Geophysical Research Letters, 35, L23710. DOI: 10.1029/2008GL035408.

Yi J, Li QF, Gu AL, Men ZH, He WX (2001). Advances on biology characteristics the rhizomatous grasses. Journal of Arid Land Resources and Environment, 15(S1), 1-16. [易 津, 李青丰, 谷安琳, 门中华, 何文兴 (2001). 根茎类 禾草生物学特性研究进展. 干旱区资源与环境, 15(S1), 1-16.]

Yue X, Zuo X, Yu Q, Xu C, Lv P, Zhang J, Knapp AK, Smith MD (2019). Response of plant functional traits of Leymus chinensis to extreme drought in Inner Mongolia grasslands. Plant Ecology, 220, 141-149.

Zeng XP, Zhao P, Cai XA, Sun GC, Peng SL (2004). Physioecological characteristics of Woonyoungia septentrionalis seedlings under various soil water conditions. Chinese Journal of Ecology, 23(2), 26-31. [曾小平, 赵平, 蔡锡 安, 孙谷畴, 彭少麟 (2004). 不同土壤水分条件下焕锯 木幼苗的生理生态特性. 生态学杂志, 23(2), 26-31.]

Zhang GT, Wang KX, Wang LX, Wang Y (2007). Couple effect of the soil water and fertility in sustainable agriculture in Liaoning. Journal of Anhui Agricultural Sciences, 35, 7531-7555. [张广涛, 汪可欣, 王丽学, 王宇 (2007). 水肥耦合技术在辽宁地区农业可持续发展中的应用分 析. 安徽农业科学, 35, 7531-7555.]

Zhang HX, Gao YZ, Tasisa BY, Baskin JM, Baskin CC, Lü XT, Zhou DW (2019). Divergent responses to water and nitrogen addition of three perennial bunchgrass species from variously degraded typical steppe in Inner Mongolia. Science of the Total Environment, 647, 1344-1350.

Zhang ZN, Wu GL, Wang D, Deng L, Hao HM, Yang Z, Shangguan ZP (2014). Plant community structure and soil moisture in the semi-arid natural grassland of the Loess Plateau. Acta Prataculturae Sinica, 23, 313-319. [张志南, 武高林, 王冬, 邓蕾, 郝红敏, 杨政, 上官周平 (2014). 黄土高原半干旱区天然草地群落结构与土壤水分关系. 草业学报, 23, 313-319.]

Zhao XF, Xu HL, Zhang P, Tu WX, Zhang QQ (2014). Effects of nutrient and water additions on plant community structure and species diversity in desert grasslands. Chinese Journal of Plant Ecology, 38, 167-177. [赵新风, 许海量, 张鹏, 涂文霞, 张青青 (2014). 养分和水分添加对荒漠 草地植物群落结构和物种多样性的影响. 植物生态学 报, 38, 167-177.]

Zhou P, Geng Y, Ma WH, He JS (2010). Linkages of functional traits among plant organs in the dominant species of the Inner Mongolia grassland, China. Chinese Journal of Plant Ecology，34，7-16. [周鹏, 耿燕，马文红，贺金生 (2010). 温带草地主要优势植物不同器官间功能性状的 关联. 植物生态学报, 34, 7-16.]

Zhu JT, Li XY, Zhang XM, Zeng FJ, Yang SG (2010). Leaf functional traits of Ceratoides latens in northern slope of Kunlun Mountain and its regional difference with the altitude. Journal of Desert Research, 30, 1325-1330. [朱军 涛, 李向义, 张希明, 曾凡江, 杨尚功 (2010). 昆仑山 北坡驼线愁叶片功能性状及其海拔差异性. 中国沙漠, 30, 1325-1330.]

Zhu TC (2004). Bioecology of Leymus chinensis. Jilin Science and Technology Press, Changchun. [祝廷成 (2004). 羊草 生物生态学. 吉林科学技术出版社, 长春.]

责任编委: 何维明 责任编辑: 李 敏 实习编辑: 赵 航 